\title{
Identifying measurement knowledge and its relationship to engineering design
}

\author{
P. Saunders ${ }^{1, \star}$, S. Giudice ${ }^{2}$, and J. Swart ${ }^{3}$ \\ 1 Department of Mechanical Engineering, University of Bath, BA2 7AY, UK \\ 2 Measurement Engineering, Rolls-Royce plc, Derby, DE24 8BJ, UK \\ 3 School of Management, University of Bath, BA2 7AY, UK
}

Received: 13 April 2014 / Accepted: 24 April 2014

\begin{abstract}
Capable measurement of the size and shape of components is a prerequisite for precision manufacturers. However, the design community may not fully consider measurement issues that could arise when components are manufactured, and the manufacturing community may not wholly appreciate the value that measurement data can bring to design. This paper investigates measurement knowledge in this relationship to find out if there are specific knowledge types that could be developed to improve the likelihood of fulfilling design intent. A proven systems-based approach was applied to an illustrative case to develop a model of knowledge flows and identify key knowledge types. For this case study, it was found that knowledge about measurement is dispersed and managed through a variety of practices which are relatively isolated from design. Furthermore, conflict was uncovered between the knowledge types, reinforcing the view that a more inclusive and integrated approach is required. In the course of the research, a novel methodology was developed in which group modelling was used to generate questions, rather than the more typical approach of grounding a systems model on interview data.
\end{abstract}

Keywords: Knowledge management; manufacturing measurement; engineering design

\section{Introduction}

What comprises measurement knowledge? By this, we refer to knowledge pertaining to measurements of size and shape that pervade manufacturing industry. In this paper we attempt to identify measurement knowledge, so that we can review its place in the design process. Additionally, we reflect on the place of design in facilitating measurement. We begin with a discussion of what it means to measure, and why we think it might be fruitful to consider the relationship between measurement and design. We then examine how the concept of know-how in action (KHiA) [1] can help us disentangle and articulate measurement knowledge. Through a case study in a precision manufacturing environment, we show how an integrative approach, known as system-based knowledge management (SBKM) [2], can be used to locate measurement KHiA. We discover what measurement KHiA comprises for this particular product creation system, and discuss the theoretical and practical implications.

\subsection{Measurement in manufacturing}

What does it mean to measure? In manufacturing, it is often understood to mean physical measurement, such

^ Correspondence: p.c.h.saunders@bath.ac.uk as determining the size and shape of various geometrical features. In this context, measurement answers questions such as: "What size is that hole?" "How round is it?" "How deep is it?" Such measurements may be performed several times on each feature for different manufacturing operations, and there could be thousands of features on each product. Other properties, such as residual stress, may be more critical in many scenarios; however, there are generally fewer kinds of these measurements to carry out, and consequently fewer opportunities for improvement. It is the geometric measurements that are the focus of this paper.

In its most direct application, measurement can be regarded as a means to confirm that the output of a manufacturing process is in accordance with design intent. The results of measurement are frequently also used to control and improve other aspects of the product creation process. For example, manufacturing processes may be modified to ensure that products are made right first time, or the results from the measurement may be used to inform the design of new products. Measurement is therefore a fundamental process that is needed within most manufacturing organisations. This has been acknowledged since the days of the Industrial Revolution, when the Victorian engineer, Joseph Whitworth, is reputed to have remarked "you can only make as well as you can measure". 


\subsection{The rationale for identifying measurement knowledge}

Many organisations attempt to reduce risk and product development time by taking account of manufacturing activities as early as possible, sometimes known as frontloading; strategies such as design for manufacture (DFM) and its counterparts, including design for quality [3,4], are prominent examples. One might expect such strategies to ease the task of measurement during manufacturing. In addition, robust design is frequently employed to reduce the impact that manufacturing variation could have on the fulfilment of design intent [5]. Yet DFM-like methodologies and the practice of robust design are insufficient for critical features which may need additional controls through high accuracy measurement; meanwhile robust designers demand increasingly accurate measurement data in order to support sophisticated analysis models [6]. As a result it seems that design and measurement are often not as well integrated as they could be [7].

The premise of this investigation is that one reason for this apparent misalignment may be that measurement processes can be too entangled with manufacturing as a whole; remarks such as "manufacturing would simply be impossible without measurement" [8] are typical when attempting to articulate the value that measurement activity brings to a system. We aim to unravel measurement knowledge with the help of a case study. We recognise that it will not be possible to generalise from the case study since the optimum mix of knowledge management practices required in a socio-technical system is highly dependent on context $[9,10]$. However, we hope that if we can find out what measurement knowledge comprises for this case, we can draw up at least one possible direction for driving alignment between the processes of design and measurement.

\section{Theory}

\subsection{What is knowledge?}

The dominant approach to categorising knowledge is to define it in relation as to whether it is tacit or explicit [11]. It is often remarked that some types of knowledge, such as tacit knowledge, cannot be written down [12]. Nonaka argued that some types of knowledge can be readily codified [13] - this is explicit knowledge. The fundamental theory of knowledge creation, for Nonaka and Takeuchi is the spiral of conversion from tacit to explicit knowledge [14]. The approach has been criticised. For example, Wilson points out that "tacit" means that something is hidden, and therefore by definition, it cannot be converted. Moreover, even if it were possible to convert some aspects of tacit knowledge, the process could be costly [15]. Nonaka et al. [16] responded to such criticisms by extending the theory to say that knowledge assets can be categorised as experiential, conceptual, systemic, or routine: experential knowledge arises from shared experiences and typically includes skills and know-how; conceptual knowledge is based on concepts, for example words or pictures that have a particular meaning to people related to an organisation; systemic knowledge is a category of knowledge that is often encapsulated in information systems, such as a product definition; whilst routine knowledge is related to the practices by which everyday activities are carried out. By mapping assets to these categories, Nonaka et al. [16] argues that the spiral of knowledge conversion can be managed appropriately; we shall refer to the output of such a mapping process as an asset map. Nonetheless, some researchers remain sceptical, believing that it is not possible to convert knowledge, and that tacit and explicit knowledge are in fact "two sides of the same coin" [17].

Alternative approaches were therefore developed, focussing on how knowledge is used in action. In the actionbased approaches, knowledge is seen to be integral to the other things that give it value [18]. Imagine the case of the designer in a manufacturing organisation who is using her expertise to create an innovative new feature. She employs her knowledge to interact with people, products, and processes to create value; a newcomer to the job might not be able to understand the nuances of what is written down and said, to achieve the same outcome. Our hypothetical designer knows she is exceptional at her job because she is actually in the process of doing it. This is an example of knowledge that Swart terms "know-how in action" (KHiA) [1]. Before moving on to consider measurement knowledge, we should note that the two approaches, that is tacit/explicit characterisation, and viewing knowledge as KHiA, can be considered to be complementary.

\subsection{Measurement knowledge as $\mathrm{KHiA}$}

If measurement is defined around physical measurements that answer questions such as "what size is that hole", measurement knowledge could be regarded as the ability to develop the processes that provide those answers. Sydenham points out that the ability to provide hard measurements, such as the size of a hole, needs to be put into its broader context [19]. Sydenham notes that few people have attempted to understand the relationship between the measurements that are taken, and their value in addressing wider critical issues [19].

The value of measurement knowledge in a manufacturing context is discussed extensively by Kunzmann et al. [20]. This source highlights that often measurement is seen as a non-value adding activity, and that the ambitions of manufacturing engineers should be to reduce inspection activities. Kunzmann et al. [20] draws on the economic argument that measurements provide information and knowledge which can be used to further optimise designs. However in order for this to be possible pre-conditions must be met beyond the acquisition of the measurement itself, which include making the information readily accessible and having the qualified people willing to interpret this. It is only when the right people receive the right information at the right time that the information can then be transformed into valuable knowledge. 
Such viewpoints are heavily biased in favour of investing and conducting measurements; the more astute reader might link this to the agenda of the authors. Therefore a counterargument might be that conducting measurements is very expensive and the investment required might not return sufficient financial benefits to build a strong business case. However, the nature of the environment in which precision manufacturing is typically carried out challenges the argument. Industries such as aerospace, defence, and nuclear power take place within a stringent regulatory framework and require measurements to ensure conformance and therefore safety. Thus the debate becomes, not one of whether to measure or not, but rather how do we extract as much value as possible from the information gleamed to create value-adding knowledge. This can only be achieved if Kunzmann et al.'s prerequisites of available information and primed people are satisfied, which leads us to an interest in the relationship between measurement and design. Since the preparedness of people is central to this relationship, we believe it appropriate to think of measurement knowledge as KHiA, whilst not losing sight of the tacit/explicit characterisation.

\subsection{How can we identify KHiA?}

Koestler [21] advises that there are essentially two complementary ways of describing the world: through reticulation (networks) or arborisation (tree-like structures). Whilst the use of tree-like structures is well-established for modelling business processes, for example through descriptive languages such as IDEF-0 [22], such structures are not necessarily well-suited for describing the dynamic behaviour inherent in socio-technical environments [23]. In these cases, network structures can be of use.

System-based knowledge management (SBKM) is a research instrument that fits into the network-centred way of thinking, and was introduced by Powell and Swart [2]. In SBKM, one first develops an influence diagram for the system under consideration. An influence diagram is a way of diagramming causal links between variables; because it can show mutual causality, it is well-suited to illustrate feedback and dynamism [24]. Having built a model for the target system, loops are identified which drive key variables (such as "profit"). The links in these loops are then reviewed to identify significant knowledge types. In this sense, a knowledge type can be defined as a group of experience-based concepts that relate closely to one another and that has an impact on the business system.

\section{Case study}

\subsection{Background}

The case study is concerned with turbine design and manufacture at Rolls-Royce plc. Turbine blades are small, yet important components in the product that has formed the basis of Rolls-Royce's success - the gas turbine engine. They are subject to a demanding environment within the engine due to their location in the gas stream directly behind the combustor. They must be engineered to function at the edge of technological capability, and consequently small improvements can have a major impact on the performance of the engine. Turbine blades are optimised through a semi-automated robust design process. Automation allows faster and more effective design iterations, taking into account knowledge from skill disciplines that include aerodynamics, thermal, stress, manufacturing, and cost. In this design process, measurement is considered within a manufacturing context.

We tested our assumption that it could be beneficial to promote the importance of measurement in the robust design process, so that design parameters can be more directly related to the quality, cost, and feasibility of measurement. Design optimisation is centred on a product lifecycle management (PLM) system, and there is a trend towards integrating an increasing number of processes through such systems [25]. Specifically, it has been argued that PLM is currently under-used with regard to measurement processes; Maropoulos and Ceglarek [26] concluded an extensive review on design verification and validation by noting that PLM has the potential to improve the line of sight between product definition and measurement capability, thereby enabling designs to be verified earlier in the lifecycle.

\subsection{Methodology}

We began by employing system-based knowledge management (SBKM) [2] to develop a model of the turbine blade creation system. SBKM draws its power from involving actors within a socio-technical context to highlight the variables that they feel are significant, and then finding relationships between these variables to identify knowledge flows. Once a model is developed the most dominant loops are extracted (for a detailed explanation see [2]). The loops are then examined from a knowledge-based perspective to identify the specific knowledge inherent in the loop.

Since the technique was new to the organisation, it was felt helpful to develop the model as a group, allowing people to become involved early to accelerate learning. Accordingly, a group was formed comprising one representative from each of the design, manufacturing, and measurement functions, as well as the programme manager who was responsible for changes to the design optimisation process. A focus group meeting was held at one of the manufacturing facilities in the UK, in a room away from disruption. After explaining the purpose of the study, we introduced the technique of SBKM, set the ground rules for model building, and went on to develop a map of the turbine blade creation system, as shown in Figure 1. This was achieved by firstly canvassing for variables which participants felt to be important in the system; these could be quantitative variables (such as "manufacturing yield") or variables of a more qualitative nature (like "workforce skills"). The facilitator captured the results directly into a software tool [27], projecting the emerging model to a 


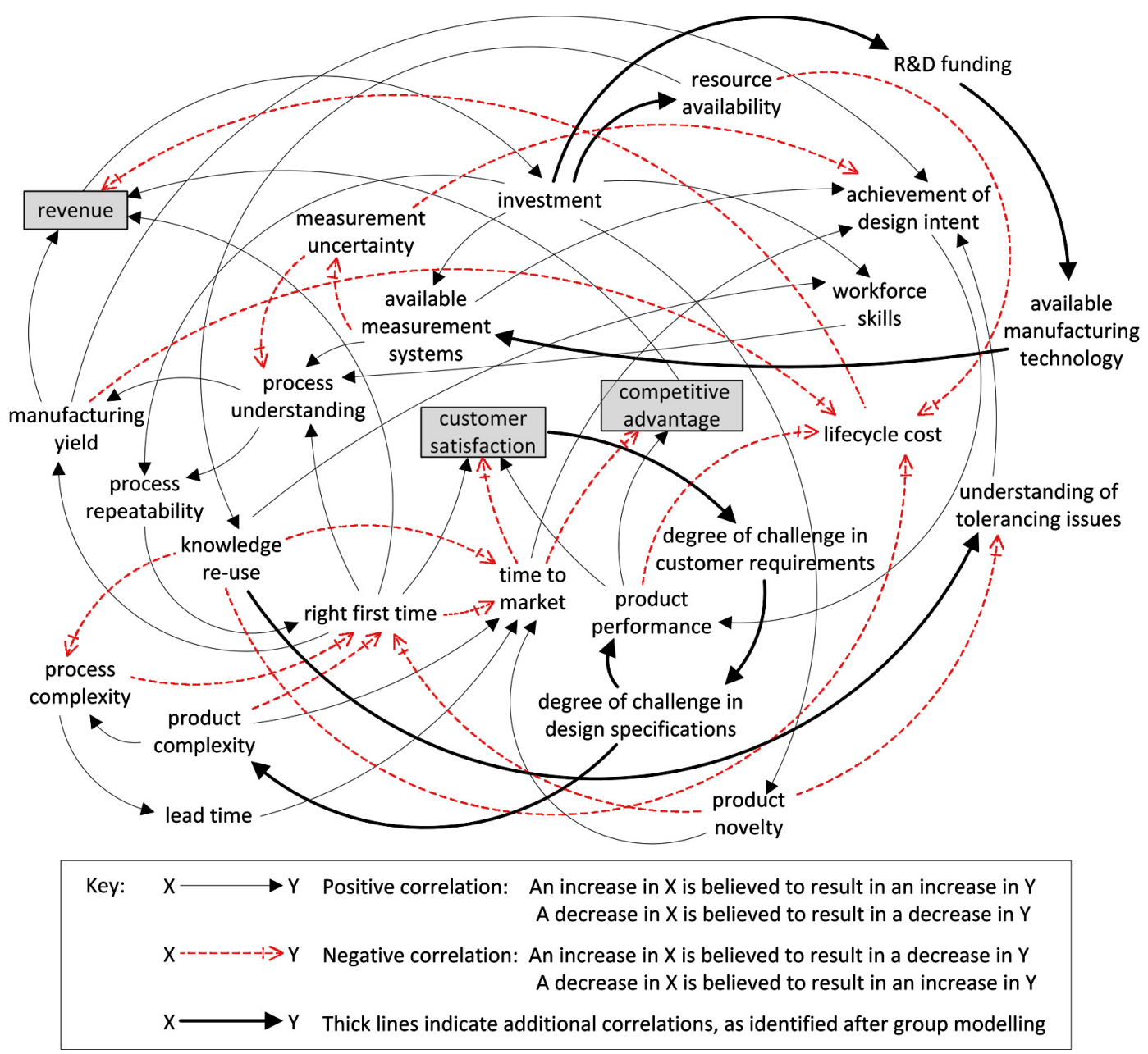

Fig. 1. Influence diagram for the turbine blade creation system.

screen, and allowing the group to reflect on the model as it grew.

Following identification of the variables, the group began to suggest links to reflect the causal relationships between them. Solid lines indicate positive correlations, whilst dashed lines show negative correlations. The effect is not necessarily strong and there may be other mechanisms at work. For example, an arrow is shown from the box labelled "revenue" (top-left on Fig. 1) to "investment" since there was a belief that there is a positive correlation between the two variables. A positive correlation implies that the group believed that an increase in revenue would result in an increase in investment; positive correlation also implies that a decrease in revenue would result in a decrease in investment. A dashed arrow can also be seen to arrive at "revenue" from "lifecycle cost"; this reflects the group's view that lifecycle cost has a negative correlation with revenue - Rolls-Royce, like most high value manufacturing companies, is incentivised to reduce the lifecycle cost of their products. Whilst some of the relationships that were identified were not contentious, such as the link between "process complexity" and "lead time", others prompted debate. For instance, although the group were unanimous in the idea that there was a correlation between resource availability and knowledge re-use, it took some time to for the group to agree whether the correlation was positive or negative. However, consensus was eventually reached on all of these points.

At the end of the meeting, the group selected "revenue", "customer satisfaction", and "competitive advantage" as the three variables which they felt to be critical to the success of the business; these are shaded grey on the diagram. Overall, the meeting took around ninety minutes and resulted in a model that could be considered the shared and representative view of the group, although it does not seek to be definitive.

Finally, during a separate consultation with the company's global measurement process owner, a small number of additional relationships were identified, as shown by the thicker lines.

\section{Findings: the content of measurement knowledge}

The final model is complex, containing twenty-six variables, fifty-six arrows, and several hundred unique loops, where a loop is a cyclic arrangement of arrows. Some of 


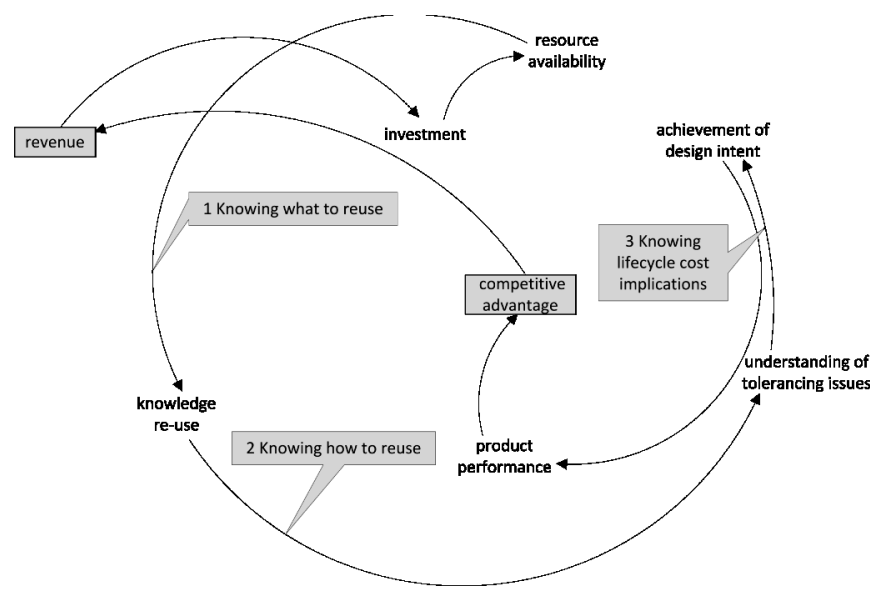

Fig. 2. Virtuous circle of standardisation.

these loops are reinforcing. A reinforcing loop has the potential to exhibit resonant behaviour in which, without any external mechanisms, variables in the loop would continue to increase or decrease depending on their initial direction. Reinforcing loops can be contrasted with goalseeking loops, which produce asymptotic behaviour; that is, without any external mechanisms, variables in the loop will tend to level out. Goal-seeking loops can be distinguished from reinforcing loops by examining the number of arrows that indicate negative correlations (the dashed lines in Fig. 1); an odd number means that the loop is goalseeking. Our motivations for both reinforcing and goalseeking loops are the same; we wish to influence them in ways that increase the key variables of revenue, customer satisfaction, and competitive advantage.

From the many loops that could have been identified, the authors isolated five that appeared to have a particularly strong impact on key variables. Although this initial choice was made independent of the group, the loops were refined with the group during a second ninety minute meeting. At this second focus group meeting, a "story" was agreed for each one. The story is a means of re-telling the loop in a verbal form. The stories help to describe the mechanisms at work within each loop, and in this instance helped the group to refine the loops to just three which they felt most important to the product creation system as they understood it; these loops relate to standardisation of measurement methods, manufacturing process understanding, and novelty, as outlined in the following sub-sections.

\subsection{Virtuous circle of standardisation}

The first loop is shown in Figure 2. This is a reinforcing loop in which there would be benefits to invigorating the loop. In this case, we could continually increase competitive advantage and revenue if positive momentum could be sustained. The wording that was agreed for the story was as follows: "As revenue increases, more resource will be expended on knowledge reuse (such as standard features and standard work) which improves understanding of tolerance issues. Improved understanding leads to a greater degree of meeting design intent. As a consequence, product performance also improves, resulting in increased competitive advantage and revenue".

By interrogating each interaction we can begin to find out what type of knowledge is important to drive each loop, and therefore identify ways of influencing the flow of the loop. Where the knowledge has been identified as relevant to the issue of sharing knowledge between design and measurement, this was annotated on the diagram.

For example, in order to make use of an increase in resource availability to ensure that that knowledge re-use is improved, one needs to know what measurement knowledge should be re-used (labelled "knowing how to reuse" in Fig. 2); it was considered that this type of knowledge is important for both design and measurement. On the other hand, the knowledge needed to gain competitive advantage from improved product performance was considered outside of the remit for measurement KHiA. The group identified three knowledge types for this loop, which can be categorised as listed below.

\section{- Knowing what to reuse}

This knowledge type refers to the ability to evaluate the utility of measurement knowledge that has been gained from experience with previous components. In the case study, coordinate measuring machines dominate the system of measurement, and experiments are carried out on the same machines that will be used in production to evaluate their capability; simulation of measurement capability is rarely used within an industrial context [28]. However, these experiments cannot be performed until prototypes have been manufactured, and the results cannot easily be generalised because they are highly sensitive to the measuring task [29]. It is therefore difficult to know whether a design change is likely to challenge the measurement system, even when employing the same technology.

- Knowing how to reuse

In addition to knowing what to reuse, one also needs to know how. When this knowledge type is strong, the design and measurement functions would have a joint understanding of why particular measurement methods have been employed in order to verify specific geometric characteristics. In the case study, it was noted that when a geometric characteristic is considered to be particularly challenging to verify, the method of measurement is made explicit within the design definition. For example, a small number of target points may be identified in the design model which strongly promotes the use of a coordinate measuring machine in discrete point mode. In order to change the measurement method to use technology that acquires substantially more measurement points at the expense of reduced single point accuracy (such as 3D structured light), a change to the design definition would be required.

It is important to note that although tolerances were explicitly identified in the variable name "understanding of tolerance issues" during group model-building, 


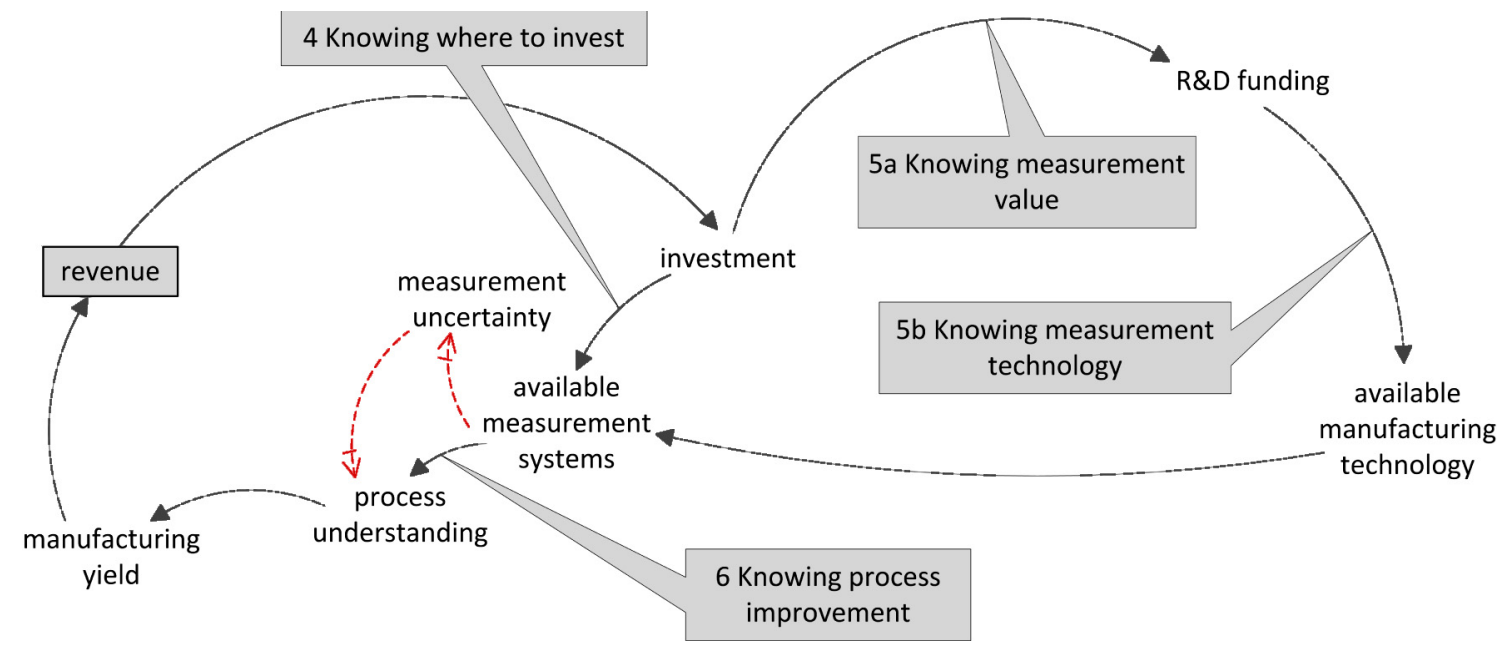

Fig. 3. Virtuous circle of process understanding.

the comment is relevant to other measurement issues, including feature accessibility, datum selection, and the existence of sufficient material on which to perform the measurement.

- Knowing lifecycle cost implications

Reduced lifecycle cost is the primary objective within our robust optimisation case study. The manufacturing cost of a turbine blade is low compared to costs associated with any detriment in engine performance, or shorter times between engine overhauls, that might result if measurement failed to spot non-conformance. As for the previous knowledge type, although tolerances were identified in one of the variable names during group model-building, the concept is applicable to other measurement issues.

\subsection{Virtuous circle of process understanding}

The second loop is shown in Figure 3. This is also a reinforcing loop that we should like to encourage since it has the potential to drive up revenue. The story that was developed for this loop is as follows: "As revenue increases, more investment will be made which should include measurement systems, both directly in the business, and by facilitating technology development. With increased availability of measurement systems, understanding of the product creation process improves. Process understanding results in increased manufacturing yield, for example, from reduced levels of final inspection".

We followed the same procedure as for the first loop, interrogating each interaction to identify key knowledge types. The knowledge types derived for this loop relate primarily to the manufacturing system and are described below.

- Knowing where to invest

In order to make use of an increase in investment to ensure increased availability of measurement systems in the business, one needs to know where to invest. In the case study, key characteristics are identified early in design and are flowed down to identify important geometric requirements. Interdisciplinary teams assess the criticality to design, manufacturability, and measurability of these requirements. Where it is considered that there is a high risk of failing to produce an important feature within specification, and where the selected manufacturing method is not trusted to consistently produce the feature within specification, measurement may be required to act as a gatekeeper. In this situation, a measurement system that provides variable data is preferable to one which gives simple yes/no answers. However, it was found that it is not always considered economically viable to acquire variable data when at the limits of measurement capability. Thus knowing where to invest is not sufficient on its own; new technology may be required.

- Knowing measurement value and knowing measurement technology

The knowledge types "knowing measurement value" and "knowing measurement technology" can be treated together as they form an alternative route to increasing the availability of measurement systems. In this instance, investment is funnelled to research and development (R\&D). Knowledge of the value that differing measurement technologies could bring to the business is needed in order to determine where R\&D effort should be directed. For R\&D funding to be translated to better measurement technology, a thorough understanding of the state of the art is required. New technology needs to be considered in the light of the complete supply chain where different measurement systems may be employed, raising questions as to how much resource the organisation should expend in influencing the supply chain to take up any new measurement technology that is developed. There is an important link between design and measurement because the design definition influences which types of measurement systems can be employed for verification, as previously discussed for "knowing how to reuse". 


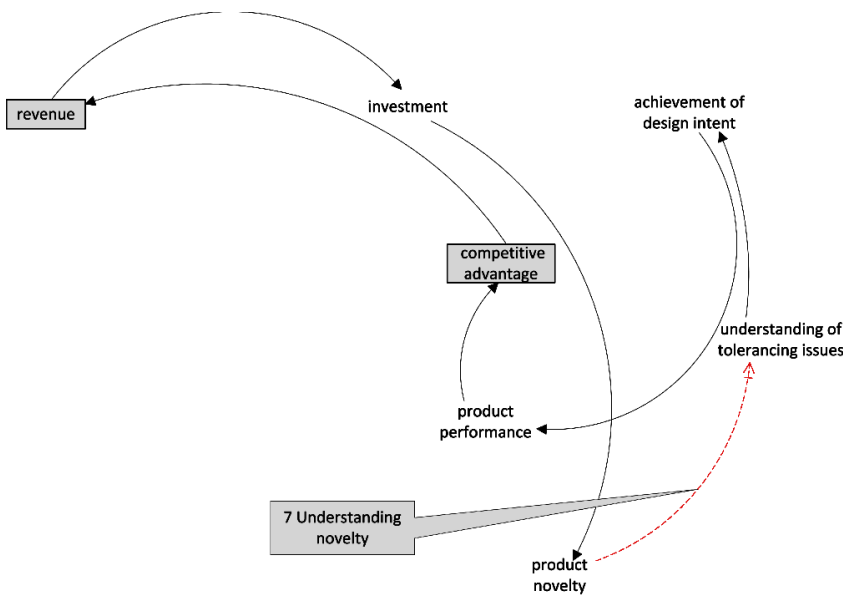

Fig. 4. Challenge of novelty.

\section{- Knowing process improvement}

The last knowledge type identified for the loop of process understanding is about knowing how to make a process improvement from the measurement systems available within the business. This was identified as a major strength within the current product creation system for turbine blades. With a strong understanding of the manufacturing process, there is the potential to reduce measurement and manufacturing cost. Concession management was identified as a potentially significant link between design and measurement. Measurement data could be used to re-run design analysis models, thus determining the impact of as-manufactured geometry on function.

\subsection{Challenge of novelty}

Finally, a goal-seeking loop was identified for product novelty, as shown in Figure 4. The story that was developed is as follows: "As product performance improves, competitive advantage is gained. This leads to an increase in revenue and subsequent increase in investment. Paradoxically, when investment is made in product development, novel features may be introduced to the product which could make it more challenging to meet the design intent".

In contrast to the reinforcing loops identified for the standardisation and process understanding loops, this is a goal-seeking loop. The additional measurement knowledge identified here is required to reduce negative consequences that could result if this knowledge type were insufficient.

- Understanding novelty:

This knowledge type refers to the need to appreciate the factors that make a new part different from a measurement perspective. In the case study, it was evident that this required considerable experience and is hard to document. The engine development cycle is decades long, and it is therefore challenging to retain the requisite knowledge within the business. However, without such understanding, a design change may demand unnecessarily complex new systems, processes, or skills in measurement. Similarly, a measurement change could have ramifications on design methods if they are reliant on measurement data. In a system where this knowledge type is strong, novelty would be detected in the early stages of design and a dialogue would ensue between design and measurement to develop good solutions.

\section{The location of measurement knowledge}

In order to better understand where measurement KHiA is currently located within the product creation system for our case study, we decided to approach a representative set of stakeholders to gather additional data. Interviews were held with an expert practitioner from each of the design, manufacturing, and measurement functions, in addition to the part owner. The representatives from manufacturing and measurement had participated in the initial group-modelling session, and all four were familiar with the objectives of the robust design process. The interviews were of approximately ninety minutes duration, at the interviewees' place of work. The interviews were not recorded, though the responses were documented in a common tabular form, allowing the interviewees to subsequently quickly review their answers. The interviews were semi-structured, building on the knowledge types that had been identified through SBKM. For each of the knowledge types, we asked four questions. The questions were aimed at: (1) seeking examples; (2) finding out how the knowledge type is currently managed; (3) eliciting views on what should happen ideally, and finally; (4) gathering alternative perspectives on what this might mean for future integration through PLM.

The examples offered by the interviewees (for the first question) were used in the previous section to help describe the knowledge types. The opinions relating to what the interviewees felt should happen, and how PLM integration should proceed (for the third and fourth questions), inform the discussion in the next section. The remainder of this section is focussed on those responses which relate to how the knowledge types are currently managed (in answer to the second question). The responses on this topic provided information about which knowledge practices are employed to manage each knowledge type. The practices have been categorised according to the type of knowledge asset which they attempt to manage: experiential, systemic, or routine - no practices were identified for the conceptual domain (definitions from [16]). The practices were then correlated with the knowledge types identified from SBKM to form the matrix structure shown in Figure 5. The intersecting cells show where an interviewee described a practice as relevant to the consumption or production of a knowledge type.

For example, the knowledge type "knowing what to reuse" was described as being partly managed through procedures and best practices by the manufacturing representative (as shown by the "P" in the intersection of the first row with the column "procedures and best practices"). All bar the design representative mentioned 


\begin{tabular}{|c|c|c|c|c|c|c|c|c|c|}
\hline & \multicolumn{9}{|c|}{ Knowledge Practices } \\
\hline & \multicolumn{2}{|c|}{ Experiential } & \multicolumn{5}{|c|}{ Systemic } & \multicolumn{2}{|c|}{ Routine } \\
\hline & 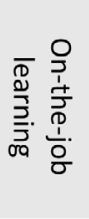 & 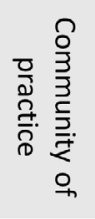 & 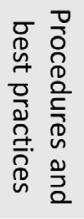 & 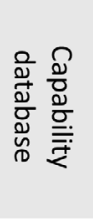 & 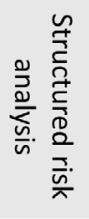 & 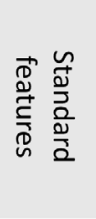 & 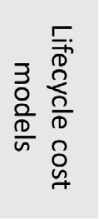 & 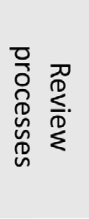 & 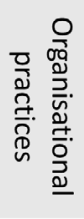 \\
\hline Knowing what to reuse & & & $\mathrm{P}$ & PMO & & & & D & \\
\hline Knowing how to reuse & & & & D & & P & & 0 & \\
\hline Knowing lifecycle cost implications & & & & & M & & DP & MO & \\
\hline Knowing where to invest & PMO & & & & D & & & MO & \\
\hline Knowing measurement value / tech & $\mathrm{P}$ & M & M & & & D & & & MO \\
\hline Knowing process improvement & & & & DPMO & & & & & \\
\hline Understanding novelty & PMO & & & & & & & & M \\
\hline
\end{tabular}

Fig. 5. Knowledge type-practice mapping.

the role of measurement capability libraries (depicted by "PMO" in "capability database"), whilst only the design representative and part owner talked about the design and manufacturing review processes (indicated by "D" and "O" in "review processes"). It is notable that when the question was turned round to ask about the knowledge type "understanding novelty", all except the design representative described this as almost solely relying on experience; the measurement representative made a further observation that novelty may sometimes not be spotted until a method of manufacture is being developed - in other words, it is part of the routine organisational practice of preparing for production; though arguably, too late. Further, the design representative did not identify any knowledge practices that are used for "understanding novelty" in the relationship between design and measurement.

The mapping in Figure 5 demonstrates the difficulty in categorising the knowledge types along the lines of experiential, conceptual, systemic, and routine. It seems that in the current system, people need to learn how to interact with all the practices in order to manage and create value from the knowledge assets [1]. The difficulty within our case study is that these measurement practices do not appear to be integrated, and sometimes not even that widely-known.

\section{Discussion}

The motivation for this research was to identify knowledge types for measurement, and explore the relationship between these and the design process. This was achieved by implementing the method known as system-based knowledge management. Using the high value manufacturing environment as a case study, seven knowledge types are now proposed, which have been placed into three categories: (1) virtuous circle of standardisation; (2) virtuous circle of process understanding, and; (3) challenge of novelty.

The knowledge types identified for the "virtuous circle of standardisation" suggest that value could be extracted if what knowledge to reuse and how to reuse it was better understood. For example, if the ability to use a specific measurement instrument is best in class, then one might minimise risk to other parts of manufacturing by reusing this knowledge instead of exploring newer opportunities.

The knowledge types identified for the "virtuous circle of process understanding" suggest that manufacturing engineers need to know what systems to buy or develop, and how they can use measurement knowledge to improve manufacturing process knowledge, with the ultimate objective of reducing final inspection.

In the "challenge of novelty", we identified a knowledge type for distinguishing novelty and how this affects manufacturability. A lack of appreciation of this knowledge type could affect progression in two ways: first, measurement engineers might tend to rely on reusing existing knowledge, and therefore not explore other opportunities; second, design engineers might not cater for the complexity of the measurement, thereby making it difficult to manufacture.

To exploit this insight, an interactional approach could be taken. This would include a consideration of culture, infrastructure, technology, and measurement (of measurement knowledge). For example, we might seek to improve the culture around measurement knowledge by encouraging a diverse membership within communities of practice, thus promoting a freer exchange of ideas. Alternatively, a formal structure that makes the management of measurement knowledge part of the job could also be implemented. This could be done by mandating the updating and use of 
"lessons learnt" $\operatorname{logs}$ at the interaction points highlighted in Figures 2-4.

Following the SBKM activity, interviews were conducted which indicated that although knowledge types can be related to each other, their locations are dispersed and managed through a variety of practices. By employing Nonaka et al.'s asset map [16] as a framework, we explored how the knowledge types interact with current knowledge practices. We found some of the practices to be isolated from the engineering design community, suggesting that greater integration between design and measurement could yield long-term benefits. Hence we agree with the viewpoint of Maropoulos and Ceglarek [26] by suggesting that PLM could improve the line of sight between product definition and measurement capability.

However, the beliefs within a manufacturing function might be hard to overturn. Traditional manufacturing key performance indicators focus on the ability to manufacture and deliver parts and assemblies to a customer. To challenge this belief requires a view of the whole product lifecycle, not just manufacturing; measurement data has inherent value within the design and development process. For example, effective robust design processes depend on the availability of manufacturing data captured from measurements. This data can be used to conduct probabilistic studies of components so that the designs are less sensitive to variability in their final operating environment. One solution might be to manage measurement data through PLM. Doing this, would enable robust designers to cater for the variability observed, which is a blend of process variability and measurement uncertainty. Therefore, the robust designers become the customers of the measurement information who convert this into valuable knowledge, thus maximising the benefits from already active processes. Still, we sympathise with the opinion of Krogstie et al. [30], who suggested that although DFM is embedded within many companies, manufacturing engineering's voice within the design process is limited. Given this, it is our belief that the needs of the measurement community would be further under-represented, as these are perceived to be non-value adding, when discussed alongside other manufacturing activities.

Nonetheless, the main limitation of current cultures is to make as few measurements as possible, and with the primary purposes of proving conformance and controlling manufacturing processes. This means that the rich data needed to conduct probabilistic analysis, for instance as needed for robust design, is often lacking. For example, to conduct a probabilistic study of a turbine blade requires much of its complex geometry to be captured. To do this might require a move away from traditional measurement techniques (such as coordinate measuring machines) to more modern alternatives (such as 3D structured light), and further investment in the capability and protocols to store this new data. Once the data is collected new tools will then be needed to convert the measurement data into a usable format, and high power computing is needed to conduct the probabilistic analysis in reasonable timeframes. Thus in the ideal world, the value of measurement data would be seen in its ability to complement existing product improvement initiatives.

The discussion is topical because the ISO/TC 213 committee is in the midst of a major overhaul of the standards that link the disciplines of design and measurement [31]. A guiding principle for these revisions from ISO is to ensure that there is a clear separation between the processes of specification and verification. According to the tenet of "duality", the designer is responsible for translating design intent into a set of geometric product specifications, and the metrologist is responsible for subsequently defining verification operators that mirror the specifications [32]. This is also the dominant working procedure in the case study. Reflecting on the knowledge types that we have found, we believe that since designers and metrologists have an imperfect understanding of one another's disciplines, it may sometimes be appropriate to develop the specification and verification processes concurrently, with substantial interdisciplinary input.

\section{Limitations}

The SBKM activity proved to be an accessible technique that quickly enabled a group of stakeholders to develop a shared understanding of the problem space. This activity was followed up with semi-structured interviews. The methodology employed was novel, since it is more common to ground a systems model on interview data rather than the other way round [33]. By delaying interviews until after the systems model was built, we found that those stakeholders who were involved in both stages were better prepared, allowing better focus than had been achieved in previous discussions on related topics.

We recognise that there are limitations to the methodology we followed. The format of using a conventional meeting to build up the initial model introduces a risk of "group think". Perhaps more importantly, by grounding interview questions in output from the qualitative systems dynamics model, there is a danger of stifling creativity, especially as this was done within the relatively insular environment of a single business unit.

In addition, although a cross-section of people participated in the analysis, there were only a small number of them. Had a different set of participants been present in the model building session, it is likely that a quite different model could have emerged; indeed depending on the diversity of stakeholder purposes, it may have proved difficult to come to an accommodation over some aspects. It is suggested that these limitations could be mitigated by running additional case studies to learn how other organisations work with measurement knowledge.

\section{Conclusions}

We began by asking the question: What comprises measurement knowledge? We found know-how in action to be a useful model for thinking about measurement knowledge, and employed the concept to systematically identify seven measurement knowledge types that relate to 
standardisation, process understanding, and novelty. The knowledge types we found require the design and measurement functions to work together to resolve potentially conflicting purposes in measurement, for which PLM support may be useful.

We recognise the difficulty in generalising findings from a single case study, and recommend that further research is carried out to determine what measurement knowledge types are important for other industries and sociotechnical contexts.

Acknowledgements. This work was supported by the Systems Centre and the EPSRC funded Industrial Doctorate Centre in Systems (Grant EP/G037353/1) and Rolls-Royce plc. The authors are also grateful to: Nick Orchard, Neil Tatman, and Prof. Paul Maropoulos for their suggestions and comments during the research; Dr. Jose Garcia and his colleagues for their assistance with the case study, and; Prof. John Powell for his involvement in the first draft and advice on employing SBKM.

\section{References}

1. J. Swart, That's why it matters: How knowing creates value, Management Learning 42, 319-332 (2011)

2. J.H. Powell, J. Swart, This is what the fuss is about: a systemic modelling for organisational knowing, J. Knowl. Manag. 9, 45-58 (2005)

3. V. Hubka, Design for Quality and Design Methodology, J. Eng. Design 3, 5-15 (1992)

4. M. Mørup, A New Design for Quality Paradigm, J. Eng. Design 3, 63-80 (1992)

5. M.S. Phadke, Quality Engineering using Robust Design (Prentice-Hall, Engelwood Cliffs, 1989)

6. A. Deshpande, The use of geometric uncertainty data in aero engine structural analysis and design, Ph.D. thesis, University of Southampton, 2013, pp. 111-138

7. I. Cristofolini, S. Filippi, C. Bandera, The role of product feature relations in a knowledge based methodology to manage design modifications for product measurability, Int. J. Prod. Res. 47, 2373-2389 (2009)

8. National Measurement Office, Rolls-Royce Industrial Case Study: 2010, http://www.bis.gov.uk/assets/nmo/ docs/nms/economic-reviews-august-2010-updates/ rrcasestudy-july-2010.pdf [Accessed 13 April 2014]

9. C. McMahon, A. Lowe, S. Culley, Knowledge management in engineering design: personalization and codification, J. Eng. Design 15, 307-325 (2004)

10. D. Wynn, J. Clarkson, Models of designing, in Design process improvement: a review of current practice, edited by J. Clarkson, C. Eckert (Springer-Verlag, London, 2004), pp. $34-59$

11. T. Ray, S. Clegg, Tacit Knowing, Communication and Power: Lessons from Japan? in Managing Knowledge, edited by S. Little, T. Ray, 2nd edn. (Sage, London, 2005), pp. 319-347

12. M. Polanyi, The Tacit Dimension (Routledge and Kegan Paul, London, 1966)

13. I. Nonaka, A dynamic theory of organizational knowledge creation, Organiz. Sci. 5, 14-37 (1994)
14. I. Nonaka, H. Takeuchi, The Knowledge-Creating Company (Oxford University Press, New York, 1995)

15. T. Wilson, The nonsense of "knowledge management", Inform. Res. 8, 1-33 (2002)

16. I. Nonaka, R. Toyama, N. Konno, SECI, Ba and Leadership: a Unified Model of Dynamic Knowledge Creation, Long Range Planning 33, 5-34 (2000)

17. H. Tsoukas, Do we really understand tacit knowledge? in: M. Easterby-Smith, in Handbook of Organizational Learning and Knowledge Management, edited by M. Lyles (Blackwell, Oxford, 2003), pp. 410-427

18. S.D.N. Cook, J.S. Brown, Bridging epistemologies: the generative dance between organizational knowledge and organizational knowing, Organiz. Sci. 10, 381-400 (1999)

19. P. Sydenham, Relationship between measurement, knowledge and advancement, Measurement 34, 3-16 (2003)

20. H. Kunzmann, T. Pfeifer, R. Schmitt, H. Schwenke, A. Weckenmann, Productive Metrology - Adding Value to Manufacture, CIRP Annal. Manufact. Technol. 54, 155-168 (2005)

21. A. Koestler, The Ghost in the Machine (Hutchinson \& Co, London, 1967)

22. NIST, Federal Information Processing Standards Publication 183 (National Institute of Standards and Technology, Gaithersburg, 1993)

23. G. Giaglis, A taxonomy of business process modeling and information systems modeling techniques, Int. J. Flexible Manufact. Syst. 13, 209-228 (2001)

24. P.M. Senge, The Fifth Discipline: The Art and Practice of the Learning Organization, 2nd edn. (Random House, London, 2006)

25. F. Demoly, X. Yan, B. Eynard, Integrated product relationships management: a model to enable concurrent product design and assembly sequence planning, J. Eng. Design 23, 544-561 (2012)

26. P. Maropoulos, D. Ceglarek, Design verification and validation in product lifecycle, CIRP Annal. Manufact. Technol. 59, 740-759 (2010)

27. Ventana Systems, Vensim, http://vensim.com [Accessed 13 April 2014]

28. N. van Gestel, Determining measurement uncertainties of feature measurements on CMMs, Ph.D. thesis, University of Leuven, 2011, pp. 37-38

29. R.G. Wilhelm, R. Hocken, H. Schwenke, Task specific uncertainty in coordinate measurement, CIRP Annal. Manufact. 50, 553-563 (2001)

30. L. Krogstie, P. Andersson, A Case Study on Reuse of Manufacturing Knowledge - Comparing Defense Practices with Automotive \& Aerospace Practices, Proc. CIRP 3, 430-435 (2012)

31. H.S. Nielsen, Recent developments in International Organization for Standardization geometrical product specification standards and strategic plans for future work, Proceedings of the Institution of Mechanical Engineers, Part B, J. Eng. Manufact. 227, 643-649 (2013)

32. ISO 17450-2, Geometric product specifications (GPS) General concepts - Part 2: Basic tenets, specifications, operators, uncertainties and ambiguities (ISO, Geneva, 2012)

33. M. Yearworth, Inductive Modelling of an Entrepreneurial System, 28th International Conference of the System Dynamics Society, 25-29 July 2010, Seoul, Korea 